\title{
The clinical importance of accurate measurement of injected doses for radionuclide myocardial perfusion imaging
}

\author{
Raymond Taillefer, MD, FRCP, ABNM ${ }^{a, b}$ \\ a Department of Radiology, Radio-oncology and Nuclear Medicine, Université de Montréal, \\ Montreal, Canada \\ ${ }^{\mathrm{b}}$ Department of Nuclear Medicine, l'Hôpital du Haut-Richelieu, Saint-Jean-sur-Richelieu, Canada
}

Received Apr 16, 2015; accepted Apr 16, 2015

doi: 10.1007/s12350-015-0153-0

\section{See related article, pp. 256-264}

With the increased utilization of computed tomography imaging and nuclear medicine procedures, especially myocardial perfusion imaging (MPI) and positron emission tomography, radiation risk has received significant attention both in the scientific community and in the public media. Radionuclide myocardial perfusion imaging studies are the most frequently performed nuclear medicine procedures in the USA and is the second largest contributor of medical exposure to the US population, after computed tomography. ${ }^{1}$ For this reason, the latest American Society of Nuclear Cardiology guidelines suggest a target dose for MPI of $9 \mathrm{mSv}$ or lower. ${ }^{2}$ Therefore, various strategies have been advocated in order to reduce the amount of ionizing radiation exposure, such as: the use of $99 \mathrm{mTc}-$ labeled MPI agents (versus 201 Thallium), reducing the amount of injected dose adjusted for patient weight, considering performing PET imaging in very obese patients, using a stress-only protocol with 99mTc-labeled radiotracers in appropriately selected patients, the use of special dedicated imaging data acquisition and/or reconstruction methods or softwares, and dedicated new imaging devices such as solid-state cameras with higher sensitivity.

\footnotetext{
Reprint requests: Raymond Taillefer, MD, FRCP, ABNM, Department of Nuclear Medicine, l'Hôpital du Haut-Richelieu, 920 boul du séminaire nord, Saint-Jean-sur-Richelieu, Canada; rtaillefer@ hotmail.com.

J Nucl Cardiol 2016;23:265-7.

$1071-3581 / \$ 34.00$

Copyright (c) 2015 American Society of Nuclear Cardiology.
}

Nuclear medicine imaging, as for other imaging modalities, is based on compromises to be made between the ideal technical approach and the constraints of the clinical environment. The three most important basic imaging parameters for high quality images in nuclear imaging are (1) high counting statistics, (2) high counting statistics and (3) high counting statistics! MPI studies with low myocardial counts are known to be prone to either false positive or false negative studies. ${ }^{3}$ The effect of the administration of a too low dose can be dramatic, especially for a rest dose injection in the course of a same-day rest-stress injection sequence protocol with a low dose $(7-10 \mathrm{mCi})$ at rest. ${ }^{4}$ However, in order to obtain cardiac images with optimal counts, the intravenous administration of a minimal dose of radiopharmaceutical is mandatory, among other various technical parameters to consider. In this particular case, the perfect "trade-off', between sufficient counts for adequate or optimal imaging and both patient and laboratory personnel reduction to radiation exposure must be made. Lowering the radiation dose while maintaining or improving image quality should be considered as an improvement in quality of care. If all possible measures are taken to minimize the risk of radiation exposure, the benefits of the diagnostic and prognostic information derived from MPI outweigh the risks related to ionizing radiations.

In order to be able to obtain an adequate measurement of the radiation exposure from a MPI study, the knowledge of the precise amount of administered radiopharmaceutical is mandatory. Since the performance of the MPI study should be governed by the principle of ALARA (As Low As Reasonably Achievable), the pre-injection calibrated activity of the administered radiopharmaceutical should be the lowest one, taking at the same time into consideration the fact that the diagnostic quality of the image data must be 
maintained with sufficient counts. In this context, it is usually accepted that the patient effectively receives the total or near-total amount of radiopharmaceutical as determined by the measurement of the administered radioactivity either by an on-site dose calibrator or as measured by the central radiopharmacy and subsequent radioactive decay calculation. However, most of the clinical laboratories only measure the pre-injection activity into the syringe and report it as the "official" dose for dosimetric consideration or calculation, assuming that this dose corresponds to the effective dose received by the patient. However, this method of calculation does not take into consideration the potential adherence of the radiotracer to the various parts of the injection system (including syringe, tubing, and 3-way stopcock). Although most of the $99 \mathrm{mTc}$-labeled radiopharmaceuticals used in nuclear medicine ${ }^{5}$ are not considered to be problematic with regard to adherence or adsorption to the syringe or tubing (except for $99 \mathrm{mTc}$-macroaggregates of albumin used for lung scanning and detection of pulmonary embolism, $99 \mathrm{mTc}-$ sulfur colloid for liver scanning and 99mTc-dimercaptosuccinic acid for renal imaging), ${ }^{6-8}$ enhanced retention of lipophilic radiopharmaceuticals such as $99 \mathrm{mTc}$-sestamibi and 99mTc-tetrofosmin has been reported in syringes made with elastomeric plunger tips. ${ }^{9-11}$ High residual radioactivity in the syringes and tubing can lead to the delivery of a suboptimal dose to the patient resulting in poor image quality, sometimes extending the image acquisition time or, worse, forcing to repeat the study with another separate injection, unnecessarily increasing the radiation dosimetry to the patient. Furthermore, the radiation exposure to the patient is falsely overestimated.

Therefore, knowledge of both the causes and the extent of adherence of $99 \mathrm{mTc}$-sestamibi to syringes and subsequent precise measurement of the actual radiopharmaceutical injected dose is important both for image quality and for more accurate determination of radiation exposure. Ideally, this problem of variable adherence should be eliminated or significantly decreased. In determining the causes of this adherence, there are many parameters and variables to consider such as: 1-variability in the specific activity of the sodium pertechnetate used for kit preparation, 2-age of the eluate from the 99Mo-generators, 3-the amount of activity of $99 \mathrm{mTc}$-sodium pertechnetate while preparing the sestamibi kits, 4-the type and size of winged infusion set and extension tubing, 5-the use of normal saline solution for flushing, 6-the number of flushing procedures, 7-the volume and the specific activity of the injected 99mTc-sestamibi dose, 8-the effect of blood drawn back into the tubing during the administration, 9-the type and size of syringe (with or without silicone lubricant), 10-the exact location of the adherence (syringe barrel, plunger, needle, saline syringe, tubing...), 11-the residence time of $99 \mathrm{mTc}$-sestamibi in a syringe, 12-the effect of prerinsing syringes with either nonradiolabeled sestamibi or with $99 \mathrm{mTc}$-sestamibi and 13-the amount of the dead-space volume of the entire injection system. Different studies have been reported on the variability of $99 \mathrm{mTc}$-sestamibi adsorption or adherence in the syringe and tubing, showing a range from $4 \%$ to $32 \% .^{10,12,13}$ This inconsistency in the degree of 99mTc-sestamibi adherence makes it "prediction" quite difficult in a clinical context, especially when using a low volume and low dose for a rest injection in a same-day rest-stress sequence protocol. For example, if the precalibrated dose is $7 \mathrm{mCi}$ and there is a $30 \%$ adherence of $99 \mathrm{mTc}$-sestamibi in the injection system, only $4.9 \mathrm{mCi}$ is actually administered to the patient, significantly increasing the risk of image degredation due to low counts image. Furthermore, this is true only if all 99mTc-sestamibi dose has been administered without any extravasation at the site of the venipuncture.

In the current issue of the Journal, Reynolds et $\mathrm{al}^{14}$ reported the results of a study evaluating the amount of residual radioactivity of $99 \mathrm{mTc}$-sestamibi in three different types of syringes (3-part lubricated and 2-part and 3 -part lubricant-free syringes) in order to verify if the adherence of $99 \mathrm{mTc}$-sestamibi (in various parts of the system) was in excess of the expected dead-space residual activity from the entire injection system. They confirmed the results of a previous study showing that the majority of adherence occurs to lubricated components (the plunger/gasket assembly and syringe barrel) of the injection system. This strongly suggests an interaction with the silicone-based lubricant used in the very commonly available 3-part syringes. Silicone is a metalloid sharing some chemical properties with $99 \mathrm{mTechnetium}$. A related chelation with sestamibi is a possible explanation. They also showed a very high variability in the post-injection residual activity for 99mTc-sestamibi injections with the 3-part lubricated syringe with a percent adherence range from $8 \%$ to $46 \%$. This variability in the adherence seems to be related to the manufacturer of the lubricated syringe with possible variation in the type or quantity of lubricant.

Given the variable and substantial adherence of $99 \mathrm{mTc}$-sestamibi in the lubricated injection systems, it is essential to establish the best procedure for accurate determination of the actual injected dose of radioparmaceutical to the patient. In the ideal clinical context, the measured pre-injection dose of radioactivity should correspond to the entirely administered dose to the patient. This is virtually impossible to achieve for several technical reasons. However, according the above mentioned study, it seems that the use of lubricant-free 
syringes may reduce the post-injection residual activity within the syringe to an amount similar to the residual activity caused by the dead-space volume of the injection system. Although this allow for a partially more accurate dose reduction, there is still the problem of inaccurate determination of the precise amount of administered activity. Given the variability of the residual activity, the best quality control procedure would be to measure both pre- and post-injection activity in syringes and the entire injection system (including tubing and needle) in each patient. This would help to determine the exact amount of radioactivity effectively received by the patient and then a better way to predict the result on the quality of the images and a more accurate assessment of the radiation absorbed dose. This could have a significant impact in a healthcare system where the tracking of patient cumulative radiation absorbed dose becomes mandatory. Over- or under-estimation of radiation exposure may have a negative outcome on the healthcare system. In the case of dose over-estimation related to a significant amount of $99 \mathrm{mTc}$-sestamibi adhering to the injection system, this will increase the likelihood of low count images and related impacts of a poor diagnostic accuracy. The strategy of systematically increasing the precalibrated 99mTc-sestamibi dose in order to compensate for its potential adherence would only partially solve the problem. The unpredictable variability of the $99 \mathrm{mTc}-$ sestamibi adherence would still cause over- or underestimation of the true administered dose.

In summary, 99mTc-sestamibi adherence to the lubricated parts of the injection systems is unpredictable and quite variable. This can have a significant impact on the accurate measurement of the radiation exposure and on the final quality of the MPI studies. Ideally, both preinjection and post-injection 99mTc-sestamibi activity should be monitored for each patient. This would assess the true administered activity. If this not feasible, the use of lubricant-free syringe and injection system may reduce the degree of adherence. However, it would be strongly advisable to periodically and systematically assess the degree of $99 \mathrm{mTc}$-sestamibi adherence and its variability as a laboratory quality control procedure, especially if a laboratory modifies the type of syringes to be used for $99 \mathrm{mTc}$-sestamibi MPI. Similar precautions should be done for $99 \mathrm{mTc}$-tetrofosmin. In this era where radiation exposure is significantly considered and efforts to decrease the dose of injected radiopharmaceutical is one of the preferred alternative (half-dose versus halftime acquisition protocols), determination of the true administered dose becomes crucial.

\section{References}

1. US Food and Drug. FDA unveils initiative to reduce unnecessary radiation exposure from medical imaging. US Food and Drug, 2010. http://www.fda.gov/News-Events/Newsroon/Pressannouncements/ucm20085:htm

2. Cerqueira MD, Allman KC, Ficaro EP, Hansen CL, Nichols KJ, Thompson RC, et al. Recommendations for reducing radiation exposure in myocardial perfusion imaging. $\mathrm{J}$ Nucl Cardiol 2010;17:709-18.

3. DePuey EG. Image artifacts. In: Iskandrian AE, Verani MS, editors. Nuclear cardiac imaging, principles and applications. New York: Oxford University Press; 2003. p. 91-105.

4. Taillefer R, Gagnon A, Laflamme L, et al. Same day injection of Tc-99m methoxy isobutyl isonitrile (hexamibi) for myocardial tomographic imaging: Comparison between rest-stress and stressrest injection sequences. Eur J Nucl Med 1989;15:113-7.

5. Mushtaq A, Rehman T, Mansur MS, Jehangir M. Adsorption of 99mTc-radiopharaceuticals onto injection vials and syringes. J Nucl Med Tecnol 2008;36:91-4.

6. Jansson BA, Goransson MB, Agren BN. Adsorption of some technetium-99m radiopharmaceuticals onto disposable plastic syringes. J Nucl Med Technol 1998;26:196-9.

7. Keskintepe D, Ozer Y. Residual radioactivity in the syringes used in injection of radiopharmaceuticals and factors affecting the amount of residua radioactivity. FABAD J Pharmacol Sci 2005;30:176-80.

8. Stopar TG, Socan A, Peitl PK. Adsorption of radiopharmaceuticals to syringes: Setting up a reliable protocol for its assessment. Nucl Med Commun 2007;28:951-5.

9. Ponto JA. Preparation and dispensing problems associated with technetium Tc-99m radiopharmaceuticals. In: Hladik E, editor. Correspondence continuing education courses for nuclear pharmacists and nuclear medicine professionals, vol. 2. Albuquerque, NM: University of New Mexico Health Sciences Center; 2004.

10. Swanson TN, Troung DT, Paulsen A, Hruska CB, O'Connor MK. Adsorption of $99 \mathrm{mTc}$-sestamibi onto plastic syringes: Evaluation of factors affecting the degree of adsorption and their impact on clinical studies. J Nuc Med Technol 2013;41:247-52.

11. Gunasekera RD, Notghi A, Mostafa AB, Harding LK. Adsorption of radiopharmaceuticals to syringes leads to lower administered activity than intended. Nucl Med Commun 2001;22:493-7.

12. Cheng K, Ngo T. Stability and adsorption of Tc-99m sestamibi in plastic syringes. J Am Pharm Assoc 2002;42:306.

13. Hurless LM, Graves MW, Mufti OI, Quinton TM. The adsorption of technetium- $99 \mathrm{~m}$ sestamibi onto three plastic syringes: An evaluation of dosage administration error. J Nucl Med 2000;41:250P-1P.

14. Reynolds S, Kikut J. Adherence of Tc-99m sestamibi to plastic syringes could complicate efforts in dose reduction in MPI SPECT. J Nucl Cardiol (current issue to be identified). 